\title{
An Introduction to achieving Industrial Applications of Wireless Passive SAW Sensors for Advanced Monitoring
}

\author{
G. Heider ${ }^{1}$ \\ 1 SENSeOR SAS, Navigator B, 505 route des Lucioles, 06560 Valbonne, France \\ contact [@] senseor.com
}

\begin{abstract}
:
Surface Acoustic Waves (SAW) is a powerful technology enabling the design of innovative wireless passive sensors for temperature, strain or pressure measurements for instance. SAW technology exploits the piezoelectric properties of the sensors' quartz substrate. Wireless SAW sensors are powered by the energy of radio waves emitted by the associated transceiver unit when remotely interrogating the sensors in real-time. Advanced interrogation methods implemented in the embedded firmware of the transceiver compute the frequency changes in the sensor response into measurements. SAW technology has been used since more than 30 years for frequency filters, like in mobile phones with high-volume production, but its use for measurement is a breakthrough and the development from "proof of concept" to industry-standard-compliant systems was a challenge. This article describes key developments to overcome these challenges, highlights SAW sensors' features for industrial monitoring and provides examples of value-added applications.
\end{abstract}

Key words: Surface Acoustic Waves (SAW), sensor, wireless, passive, monitoring

\section{Introduction}

The need of additional remote monitoring solutions increases as industrial equipment and processes need to become more and more efficient and secure. For controlling critical parameters like temperature, on a real-time basis, even in the most remote locations or at the heart of the machinery, advanced innovative sensors like SAW sensors are essential. As their features become more widely known, the industrial applications expand. Being wireless, these sensors avoid the costs of cables and their installation. Most important, SAW sensors can often be installed right on the most critical parts of the equipment, even on rotating elements. The installation is also possible on existing equipment. Being totally passive, the sensors don't require maintenance and operate in harsh environments - where active electronics or batteries, for example, could not survive. Once installed and commissioned, the sensors operate without maintenance, featuring infinite autonomy. Industrial usage of SAW technology though requires high robustness of the system, at each level of the measurement chain: sensitive element, packaging, antenna, electronic hardware and firmware, interrogation methods, Radio-Frequency link, among other criteria.

\section{SAW Technology for Sensing}

Besides the use of Surface Acoustic Waves for the widely known and used frequency filters, the same technology can be used for sensing any physical quantity.

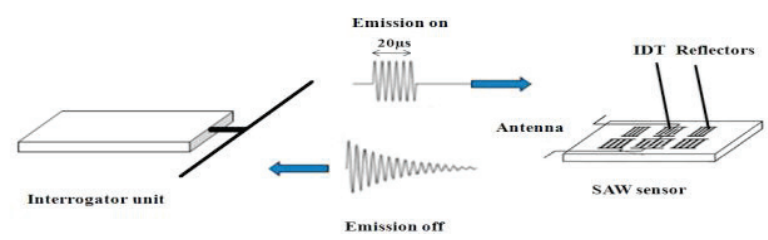

Fig. 1. Operating principle

One way to use Surface Acoustic Waves for sensing is based on the interrogation of resonators, by a transceiver through a radiofrequency link (RADAR-like interrogation). The interrogation unit sends an un-modulated RF signal in order to load the SAW sensor resonator. The incoming RF wave is converted into a surface acoustic wave (SAW) on the sensor quartz substrate due to the piezoelectricity effect. 
The velocity of a surface acoustic wave is very sensitive to the surface state. Under the effect of the physical parameter which is sensed, the velocity is modified and can be measured in the response transmitted back to the transceiver.

As the operation is based on the measurement of a frequency change on the interrogated substrate, this technology offers a wide array of possible applications: measurement of surface impact due to compression or expansion as is caused by temperature changes or physical impact - or measurement of frequency changes due to mass loading as used for chemical and biological sensing.

This technology brings major easily identified advantages like the absence of cable and the related cost reductions and easiness in commissioning, the absence of battery, avoiding maintenance visits and disposal of batteries, and the possibility to embed the sensors into material like concrete or rubber and interrogate them remotely, even in the most inaccessible places.

The wide spread availability of high volume production capacity for the SAW substrate (using the same processes as the production of SAW filters) offers high quality and maturity and allows to target low costs.

SAW sensors indicate the measurement value via frequency (in contrast to resistive or capacitive changes). Knowing that we can measure frequency changes with unparalleled high precision allows forecasting use of this technology for high precision applications. This is further supported by the characteristics of the base material - quartz - which is very stable over time.

Please Note that a SAW sensor can also be used in a wired configuration.

\section{Wired Interrogation of SAW Sensors}

The relative frequency stability $S$ of a SAWbased oscillator is typically in the $10^{\wedge}\{-9\}$ range in a 1-10 s window, rising at longer integration times due to the temperature fluctuations of the resonator (see Fig. 2).

Assuming a temperature sensitivity $\mathrm{T}$ of the transducer in the tens of $\mathrm{ppm} / \mathrm{K}$, the temperature resolution based on such a short term stability is in the $\mathrm{S} / \mathrm{T}=100 \mu \mathrm{K}$ range. This performance opens important perspectives towards achieving resolutions beyond those achievable with today's resistive sensor technologies.

Today's accuracy is in the tens of $\mathrm{mK}$ range (simple demonstrator). AQP packaging and use of a stable reference clock is seen as a promise to achieve new and long-term accuracy performance limits. Ref [1]

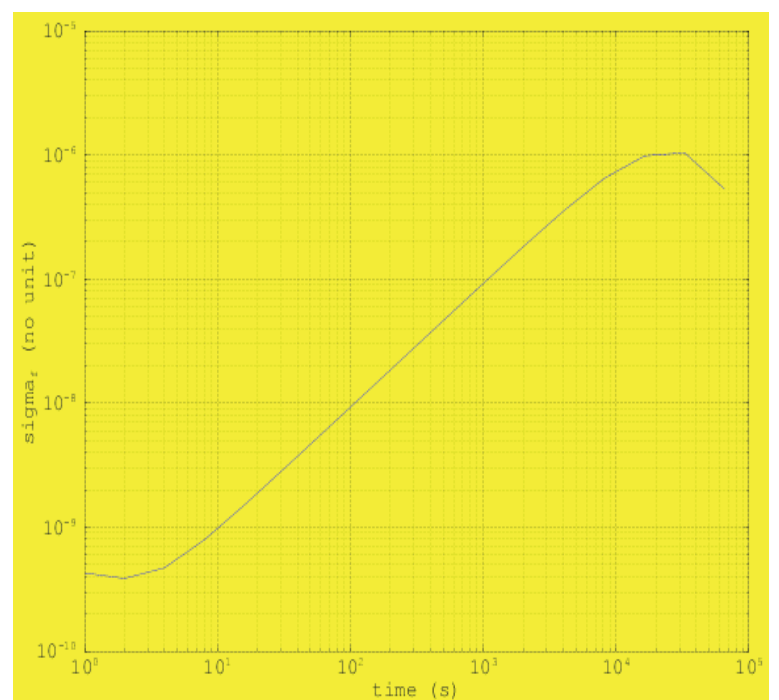

Fig. 2. Allen Variance of a wired quartz resonator

\section{Wireless Interrogation of SAW Sensors}

Wireless temperature sensing through a 434 $\mathrm{MHz}$ SAW resonator using our "Fixed comb" interrogation strategy will typically achieve a $60 \mathrm{~Hz}$ frequency identification resolution. Assuming a sensor design with a temperature sensitivity of $5 \mathrm{kHz} / \mathrm{K}$, the resolution is in the 12 $\mathrm{mK}$ range.

The actual accuracy of the measurement is related to the calibration capability, long-term drift and the actual wireless communication channel (see also below). Assuming above sensor design we typically reach $\pm 1 \mathrm{~K}$ accuracy at a temperature range of $180^{\circ} \mathrm{C}$.

Different interrogation methods allow optimization of performances per application:

- "Closed Loop" approach combining higher refresh rate, improved accuracy with respect to the fixed comb approach, and improved interrogation range with respect to the 2-point (1-point) methods $(350 \mathrm{~Hz}$ refresh rate @ 8 averages)

- "FM 2 point" to achieve a 10 -fold resolution improvement (down to $2 \mathrm{~Hz}$ demonstrated).

- "1 point" - achieving very high refresh rate 60 us per resonance $(17 \mathrm{kHz} @ 1$ average theoretical, $8 \mathrm{kHz} @ 1$ average practical)

- "Radiomodem" - using high-volume offthe-shelf components to achieve very low power consumption, low prices and enable participation in wireless networks at the same time.

See also Ref [2] 


\section{Critical Success Factors for Wireless Interrogation of SAW Sensors}

The big difference with 'standard' wireless communication is that here we have no 'logic' on the sensor - the communication is not encoded we work directly with the 'carrier' (frequency, amplitude \& phase)!

This is important to be understood. We had to develop a lot of very advanced capabilities in order to ensure dependable operation and quality of our systems in order to cover:

- Frequency pulling - i.e. the signal is influenced by even minor changes in its environment.

- Reflections - especially in metallic cavities.

- RF Performance adaptation \& tuning to different situations (distance, rotation, etc.).

- Advanced filtering to allow operation in industrial environments and despite interferers.

- Semi-automatic operations.

- Calibration-free sensors for high volume applications.

- RF Certification.

\section{Use of differential sensors to achieve stable precision}

If environmental conditions vary, antenna impedance will also vary and consequently resonant frequencies will also vary (this applies also to aging effects and other factors influencing calibration and performance).
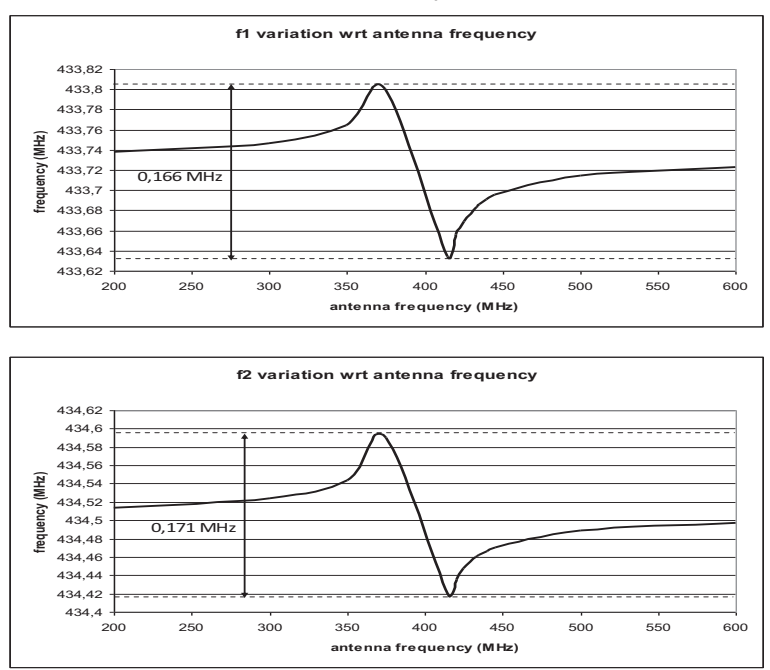

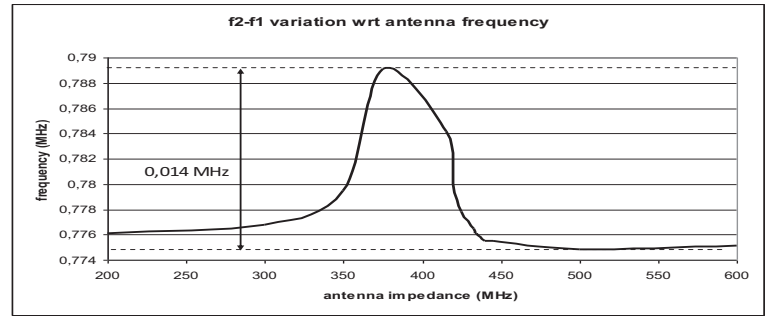

Fig. 3. Differential measurement improvement

As you can see from the 3 illustrations above (Fig.3), comparing individual resonator stability in a simulated, realistic industrial environment, using a differential approach results in a 15-times reduction of the error influence.

Only differential sensors provide the basis to contain the frequency pulling effects.

\section{Application of SAW Wireless Temperature Sensing on the crankshaft of naval Engines}

The reference application for SAW sensing technology for almost a decade now is delivered by the Norwegian Ship Industry specialist Kongsberg Maritime. Over 4000 sensors per year are installed in the crankshaft bearings of the engines. Since installation they work failure-free - despite use in very harsh conditions.

These sensors provide predictive warning of crankshaft bearing problems - hence allow the engine to be stopped for maintenance before serious damage occurs. Comparative analysis show that this system is more effective than the legacy system based on oil-mist detection as it provides alarm indication well before the main bearing temperatures rises too much causing damages.

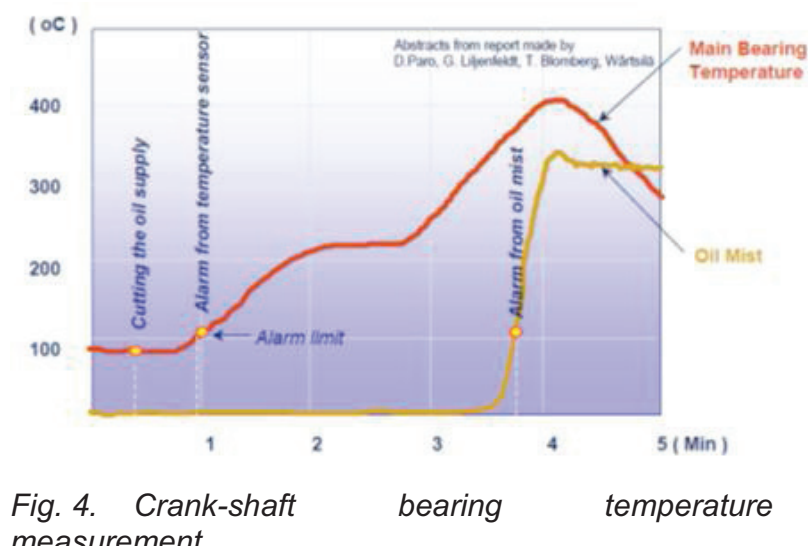
measurement 


\section{Application example of SAW Wireless Temperature Sensing in the Energy Sector}

Another value-added application of temperature monitoring with SAW sensors gaining pace is electrical equipment surveillance. With increasing energy needs, systemic overload on the power grid due to the multiplying energy sources, the equipment is used to its limits, often in a run-tofail approach. But in a smart grid, with energy efficiency improvements needed, remote continuous monitoring is a solution to enhanced performance reducing margins, while increasing safety. Temperature is a key parameter to monitor, as a temperature raise in electrical equipment is one of the key indicators of potential failure and abnormal operation. As SAW sensors are totally passive, and robust, they withstand high current and voltage, without any risk of arcing and without damage. For this, they need to be packaged in specifically designed housings, low-profile and RF-wise optimized. For instance, SENSeOR's SAW sensors have been tested up to $545 \mathrm{kV}$ and $4000 \mathrm{~A}$. They thus enable to monitor the most critical hot points on transmission lines, in transformers, in AIS switchgears for instance (on circuit breaker, on busbars). The negative effect of metallic enclosure on RF link is overcome in these latest applications by the optimized design of antennas and robust, specific interrogation methods.

Like for diesel engines, SAW sensors can monitor inner temperature of generators and turbines for instance on bearings in cylinders, on piston head, on exhaust string. The benefits are clear in all these applications: stop the equipment proactively, only when necessary, but before it's too late, enabling just-in-time preventive maintenance, enhanced performance and reduced life cycle-costs.

\section{Application example of Wireless Temperature Sensing with SAW Sensors in Cookware}

Interesting valued-added applications have also been identified in cookware and appliances. Indeed, the wireless and passive operation of the sensors enables to design wireless food probes for instance, both for application during cooking in high temperatures, and during freezing. Standard food probes are already used by professionals in ovens for instance, but the wire is always a limit for convenient use and cleaning. It often gets cut by the oven door for instance, leading to the replacement of the probe. Also, active wireless probes with battery overcome this limit, but are limited in operation to the temperatures withstood by the battery, restricted both in high and low temperature. SAW sensors ranging from $-40^{\circ} \mathrm{C}$ (or even lower down to cryogenic applications) to $165^{\circ} \mathrm{C}$ cover the needed range for freezing to cooking, while getting rid of wires and batteries. This guarantees to monitor the goods temperature all over the cooking process, eliminating possible cold chain rupture and health risks due to under-cooking or over-frying for instance. Here again, the effects of metallic cavities like ovens or fridges on the radio-frequency link need to be compensated by optimized interrogation methods in transceiver firmware.

\section{Conclusion}

We have listed some of the many possible monitoring applications enabled by SAW sensors, thanks to their unique properties, and the challenges raised by their industrial use. The wireless interrogation for instance, is one of the key advantages, but requires optimized, robust interrogation methods and antennas to achieve the required fault-free operation in harsh industrial environments.

\section{References}

Ref [1]: B. Francois, G. Martin, P. Grosclaude, M. Lamothe, G. Goavec-Merou, S. Ballandras, J.-M Friedt, Fabrication and characterization of a SAW-oscillator-based sensing system including an integrated reciprocal counter and a wireless ZigBee transmission system Proc. Ultrasonics Symposium (IUS), 2010 IEEE (1290--1293)

Ref [2]: C. Droit, G. Martin, S. Ballandras, J.-M Friedt A frequency modulated wireless interrogation system exploiting narrowband acoustic resonator for remote physical quantity measurement Rev. Sci Instrum. Vol 81, Issue 5, 056103 (2010) 